Serie e.Med Teil 3: e.News

\section{Stets auf dem neuesten Stand}

Nachrichtenportale boomen. Denn wer sich heute informieren will, wartet nur ungern auf die Berichterstattung von morgen. Schön, dass es diesen Service auch speziell für Ärzte gibt. Unter www.springermedzin.de finden Sie alles, was Sie an Neuigkeiten aus Berufspolitik, Wirtschaft und Forschung wissen müssen - schnell und unkompliziert.

W er die Ärztezeitung und die zahlreichen Magazine von Springer Medizin kennt, schätzt die gut und zuverlässig recherchierten Nachrichten. Doch der Wert einer Fachinformation ergibt sich längst nicht mehr alleine aus ihrem Inhalt. Was zählt, ist die Verfügbarkeit - und in dieser Beziehung ist das Internet unschlagbar.

\section{Journalistische Qualität + \\ Aktualität $=$ e.News}

Aus der Verbindung von qualitativ hochwertigem Journalismus und der Aktualität des Internet wird aus dem bekannten Nachrichtenangebot von Springer Medizin „e.News", die schnellste und einfachste Art, sich über alle Ereignisse im Gesundheitswesen zu informieren. Das gilt für gesundheitspolitische Nachrichten genauso wie für Neuigkeiten aus der Medizin. Alles, was sich auf kleinen und großen Kongressen, in der Forschung oder in Klinik und Praxis tut, wird in den Fachredaktionen von Springer Medizin gesichtet und aufbereitet. Als Abonnent von e.Med finden Sie darüber hinaus Hintergrundberichte, Inter- views mit Meinungsbildnern oder Videobeiträge von wichtigen Veranstaltungen. Und damit Sie wirklich nichts verpassen, screenen und bewerten die Redaktionen die internationale Fachliteratur und verfolgen für Sie die Diskussionen in der Publikumspresse. So entstehen täglich über 20 neue Nachrichten auf dem umfassendsten deutschsprachigen Medizinportal.

\section{So behalten Sie den Überblick}

Damit Sie jederzeit den Überblick behalten, können Sie die e.News nach Stichwörtern durchsuchen oder nach Fachgebieten kategorisieren. In der Rubrik „Gesundheitspolitik “beispielsweise können Sie - wenn Sie wollen - die Inhalte nach folgenden Kriterien filtern:

- Neues aus der Hauptstadt

—Aus den Regionen

_Gesundheitspolitik international

-Gesundheitswirtschaft

Und sollte es einmal von Belang sein, lässt sich auch die Chronologie bestimmter Entwicklungen problemlos nachverfolgen, indem Sie die "Treffer" nach deren Aktualität sortieren.
e.Med-Paket

Teil 1: e.Akademie

Teil 2: e.Bibliothek

Teil 3: e.News

Teil 4: e.Praxis

Eine komfortable Hilfestellung bei der großen Menge an Informationen bieten die „Dossiers“. Um Ihnen die Sortierung zu erleichtern, hat die Redaktion die News zu bestimmten Themen bereits zusammengefasst. Eine Liste innerhalb der Navigation zeigt an, zu welchen Themen eine solche "Sammelmappe" vorhanden ist.

\section{Newsletter aus über 20 Fachgebieten}

Für alle, die gar nicht erst suchen wollen, bietet sich eine weitere attraktive Möglichkeit, auf dem Laufenden zu bleiben. Die Newsletter von Springer Medizin liefern Ihnen alle zwei Wochen das Aktuellste aus „Politik \& Gesellschaft", „Praxis \& Wirtschaft" und/oder den verschiedenen medizinischen Fächern direkt auf den Bildschirm. Das „update" aus der Allgemeinmedizin, Kardiologie, Neurologie und Onkologie erscheint sogar wöchentlich.

Bestellen können Sie die Newsletter über das Registrierungsportal von Springer Medizin (http://registrierung. springermedizin.de). Dort kann man sich auch einen Überblick über das vielfältige Newsletter-Angebot verschaffen.

Bei Fragen rund um e.Med, e.News und die „updates" wenden Sie sich an unseren Kundenservice: Telefon 0800 7780777 oder kundenservice@springermedizin.de.

\title{
Ärzte Zeitung digital: Heute lesen, was morgen in der Zeitung steht
}

Im Publikumsbereich erfreuen sich AppAusgaben von Zeitungen und Zeitschriften wachsender Beliebtheit. Ab sofort ist auch Deutschlands einzige Tageszeitung für Ärzte in diesem Format erhältlich: die „Ärzte Zeitung digital“. Die App bietet gesundheitspolitische Nachrichten, Kommentare, Hintergründe zu Medizin und wirtschaftlichen Themen rund um die Praxis - im übersichtlichen und intuitiven Lesemodus, optimiert für das iPad. Die Vorteile für Leser der App-Ausgabe der "Ärzte Zeitung":

_Die Ausgabe ist schon am Vorabend verfügbar, Leser können damit die Nachrichten des kommenden Tages bequem auf dem Sofa empfangen.
- Wichtige Nachrichten werden als „Breaking News" direkt auf das Tablet geleitet und lassen sich über die sogenannte Newslasche anzeigen.

_ Die App ist so konzipiert, dass die gesamte Ausgabe im Hintergrund heruntergeladen werden kann. Anders als im Internet ist es für Leser dann möglich, sich offline durch die Ausgabe zu "blättern", ohne Wartezeiten beim Durchklicken auf einer Website.

_ Die alten Ausgaben bleiben zwei Wochen auf dem iPad verfügbar.

„Ärzte Zeitung digital“ ist Teil des e.MedPakets von Springer Medizin. Zugänglich ist die App über den iTunes-Store von Apple - allerdings nur für registrierte Nutzer von Springer Medizin, die e.Med für 30 Tage kostenlos testen wollen, oder für e. Med-Abonnenten - und zwar ohne weitere Zusatzkosten. Die Version für AndroidTablets wird Anfang 2013 verfügbar sein.

Anmeldung zum kostenlosen e.MedTestzugang: www.springermedizin.de/ eMed/Mehr Info zur App „Ärzte Zeitung digital“" www.springermedizin.de/tablet 